\title{
The HLA groups and their relationship with clinical features in Turkish children and adolescents with celiac disease
}

\author{
Necati Balamtekin ${ }^{1 \oplus}$, Gökhan Baysoy ${ }^{1 \oplus}$, Çağman Tan ${ }^{2 \oplus}$, Nuray Uslu Kızılkan ${ }^{1 \oplus,}$ \\ Hülya Demir ${ }^{1 \oplus}$, İnci Nur Saltık Temizel ${ }^{1 \oplus}$, Hasan Özen ${ }^{1 \oplus}$, Aysel Yüce ${ }^{1 \oplus,}$ \\ İlhan Tezcan ${ }^{2 \oplus}$, Figen Gürakan ${ }^{1 \odot}$ \\ Divisions of ${ }^{1}$ Pediatric Gastroenterology, Hepatology and Nutrition, ${ }^{2}$ Pediatric Immunology, Hacettepe University Faculty of Medicine, \\ Ankara, Turkey.
}

\begin{abstract}
Background. We aimed to investigate the relationship between human leukocyte antigens (HLA)-groups and clinical features, and degree of intestinal injury in children with celiac disease (CD).

Methods. Study group included 73 (50 females, 68.5\%) children with CD. Demographic and clinical features, accompanying autoimmune diseases, family history for $\mathrm{CD}$ and degree of damage in small intestinal mucosa (according to Marsh classification) at the time of diagnosis were determined. Twenty-two siblings of celiac patients without $\mathrm{CD}$ (15 females, 65.2\%) consisted control group 1, and 66 (40 females, 60.6\%) people from the normal population consisted control group 2.

Results. The allele frequencies of HLA B8, B50, C6, C7, DR3, DR7, DQ2, and DR3 homozygosity were higher in the patient group. HLA DQ2 positivity was $89 \%$ in the patient group, 73.9 and $45.5 \%$ in control groups 1 and 2 , respectively ( $\mathrm{p}<0.0001$ ). HLA A30, C14, DR11, DQ3 frequency were lower in patients compared to both control groups. HLA-DR15 alleles in patient and control group 1 was significantly lower compared to the general population ( $\mathrm{p}<0.05)$. Thirty $(41.1 \%)$ patients had typical, $43(58.9 \%)$ patients had atypical presentation. Thirteen (17.8\%) patients had other autoimmune diseases. There was no association between coexisting autoimmune diseases and the HLA antigens. Fifteen patients $(20.5 \%)$ had a positive family history for CD; patients with HLA A69, B41 and C12 alleles had a higher positive family history $(\mathrm{p}<0.05)$. Intestinal mucosal damage was as follows: 5 patients (6.8\%) had Marsh 2, 25 (34.3\%) Marsh 3a, 28 (38.4\%) Marsh 3b, 15 (20.5\%) Marsh 3c. Patients with HLA-DR15 alleles had more frequent Marsh 3a lesions ( $\mathrm{p}<0.05)$.
\end{abstract}

Conclusions. B8, B50, C6, C7, DR3, DR7, DR3/DR3, DQ2 alleles were risk factors for CD in the Turkish population. HLA C14, DR11, DR15, and DQ3 alleles were found to have a protective role in the same population.

Key words: celiac disease, histopathology, HLA typing, pediatric, Turkish.

Celiac disease is an autoimmune disease that is triggered by gluten (found in cereals like wheat and barley) intake in gluten sensitive

\footnotetext{
Necati Balamtekin

drbalamtekin@yahoo.com
}

Received 30th June 2020, revised 10th July 2020,

21st July 2020, accepted 22nd July 2020.

This study was presented as oral presentation at the 29th Turkish Gastroenterology Week Congress, 20-25 November 2012 Ankara, Turkey. individuals, eventually resulting in small intestinal impairment.

Celiac disease can present with various clinical manifestations from asymptomatic disease to celiac crisis. ${ }^{1}$ Genetic and environmental factors together play a role in disease development. HLA are known as the most important genetic factors. Celiac disease's physiopathologic mechanism is similar to other autoimmune diseases like type-1 diabetes mellitus (DM), autoimmune 
thyroiditis and Sjögren's syndrome. Celiac coexistence with these diseases is common. ${ }^{2,3}$

The purpose of this study was to determine Turkish children's HLA subgroups and to investigate the relationship between disease presentation pattern, association with other autoimmune diseases, family history of CD, severity of histopathological involvement and HLA antigen groups.

\section{Material and Methods}

This study was carried out at Hacettepe University, Faculty of Medicine, Department of Pediatric Gastroenterology Hepatology and Nutrition between January 2009 and March 2010.

We determined 73 celiac patients (study group) on follow-up diagnosed according to modified European Society for Paediatric Gastroenterology, Hepatology and Nutrition (ESPGHAN) criteria. ${ }^{4}$ Records of the patients were reviewed and complaints, demographic features, family history, presence of any comorbidities, presence of any chromosomal diseases such as Down syndrome and Turner syndrome, antibody titers, and histopathological findings (Marsh classification) of small intestine were recorded.

Additionally, two control groups were created and their HLA groups were also determined; these were $\mathrm{CD}$ patients' siblings without any health problems together with normal serum levels of $\operatorname{IgA}$ and negative celiac antibodies (control group 1) and 66 healthy individuals from the general population (control group 2).

Patients were divided into three groups according to their clinical characteristics at time of diagnosis: typical, atypical and silent celiac disease. ${ }^{4}$ Antiendomysial antibody (EMA) levels were measured by indirect immunofluorescence technique (monkey liver tissue, European Gmbh, Lübeck, Germany). Levels of tissue transglutaminase antibody
(TTG) IgA and IgG were measured by ELISA (Euroimmune, Lübeck, Germany).

All patients underwent esophagogastroduodenoscopy and six biopsy samples were obtained from duodenum and at least two of them were from the bulbus. Severity of small intestine involvement was scaled as Marsh 1, 2, 3a, 3b, 3c based on modified Marsh classification. ${ }^{5}$

Four ml of venous blood samples were taken into EDTA tubes from all patients and those in control groups after obtaining the children's and/or their parents' consent. DNA samples were obtained from blood samples by using Qiagen EZ 1 DNA Blood Kit (Qiagen Pty Ltd, Australia) and were kept at $-80{ }^{\circ} \mathrm{C}$ until HLA group study was performed. DNA samples were amplified by Olerup SSPTM DQ-DR-SSP Combi Tray (Olerup SSP AB, Sweden) and Corbett Research SSKT 011 Thermal Cycler. After agarose gel electrophoresis, bands with positive PCR products were selected and analyzed with SCORE 97 3-301 T program.

HLA A allele from one patient, HLA B allele from three patients and HLA A allele from one child in the control group couldn't be obtained because of technical problems.

This study was approved by Hacettepe University Non-interventional Clinical Researches Ethics Board (16.07.2009/ HEK 09/179).

\section{Statistical analysis}

Study data were analyzed using SPSS v.11.5 software package. Descriptive data were expressed as the mean ( \pm standard deviation) or percentage of the study group. Differences between the frequencies among groups were analyzed by chi-square or Fisher's exact test, where appropriate. Odds ratios was calculated to determine the association between the development of celiac disease and HLA types. A two-tailed p-value less than 0.05 was considered statistically significant. 


\section{Results}

The study group included 73 celiac patients [mean age $7.29 \pm 4.50$ years (range $0.71-17.40$ ); 50 females (68.5\%)]. Control group 1 included 23 patients [mean age $8.91 \pm 3.70$ years (range 3-17); 15 females (65.2\%)], and control group 2 was composed of 66 individuals [mean age $24.07 \pm 1.50$ years (range 2-61); 40 females $(60.6 \%)]$.

Rate of HLA DQ2 positivity was $89 \%$ in the patient group while it was $45.5 \%$ in control group 2 ( $\mathrm{p}<0.0001)$. Positive rate of DQ2 was higher among patient's relatives $(73.9 \%)$ in comparison to the general population, too $(\mathrm{p}=0.018)$. Although HLA DQ2 positivity rate was higher in patients compared to control group 1, it was not statistically significant $(p=0.073)$. We did not detect DQ8 allele in either patient or control groups. It was determined that frequency of HLA B8, B50, C6, C7, DR3 and DR7 alleles was significantly higher among patients in comparison to the control groups. However, the percentages of DQ2, C6, C7, and DR3 were not statistically different between patients and control group1. The frequency of HLA B27, C14, DR11, and DQ3 alleles were significantly different among groups and HLA A30 frequency nearly reached significance. Although it was not statistically significant, frequency of HLA DR15 allele in patients and control group 1 was lower in comparison to control group 2 (Table I).

Table II shows the frequencies of the combination of risky alleles (DQ2, DR3, B8) associated with CD. HLA B8/DQ2 and HLA B8/DR3 haplotypes were significantly more common in patients compared not only to control group 2 but also

Table I. Frequency of different HLA alleles in patient and control groups.

\begin{tabular}{|c|c|c|c|c|}
\hline HLA & $\begin{array}{l}\text { Patients } \\
\text { N (\%) }\end{array}$ & $\begin{array}{c}\text { Control group } 1 \\
\mathrm{~N}(\%)\end{array}$ & $\begin{array}{c}\text { Control group } 2 \\
\mathrm{~N}(\%)\end{array}$ & $\mathrm{p}$ \\
\hline \multicolumn{5}{|c|}{ Alleles more common in patients } \\
\hline DQ2 & $65(89.0)^{*}$ & $17(73.9)^{*}$ & $30(45.5)$ & $<0.001$ \\
\hline B8 & $27(38.6)^{\mathrm{a} \#}$ & $3(13.0)^{\#}$ & $8(12.1)$ & 0.001 \\
\hline B50 & $16(22.9)^{\mathrm{a} \dagger}$ & $1(4.3)^{\dagger}$ & $2(3.0)$ & 0.001 \\
\hline C6 & $26(35.6)^{\ddagger}$ & $4(17.4)^{\ddagger}$ & $11(16.7)$ & 0.024 \\
\hline $\mathrm{C} 7$ & $36(49.3)^{\mathbb{I I}}$ & $7(30.4)^{\mathbb{T}}$ & $18(27.3)$ & 0.021 \\
\hline DR3 & $58(79.5)^{\beta}$ & $17(73.9)^{\beta}$ & $23(34.8)$ & $<0.001$ \\
\hline DR7 & $19(26.0)^{\gamma}$ & $1(4.3)^{\gamma}$ & $10(15.2)$ & 0.04 \\
\hline \multicolumn{5}{|c|}{ Alleles less common in patients } \\
\hline A30 & $2(2.8)^{\mathrm{b}}$ & $3(13.6)^{b}$ & $9(13.8)$ & 0.053 \\
\hline B27 & 0 & $4(17.4)$ & $3(4.5)$ & 0.002 \\
\hline C14 & $1(1.4)$ & $7(30.4)$ & $12(18.2)$ & $<0.001$ \\
\hline DR11 & $8(11.0)$ & $7(30.4)$ & $22(33.3)$ & 0.005 \\
\hline DQ3 & $28(38.9)$ & $13(56.5)$ & $41(62.1)$ & 0.017 \\
\hline \multicolumn{5}{|c|}{ Alleles less common both in patients and control group 1} \\
\hline DR15 & $4(5.5)$ & $2(8.7)$ & $12(18.2)$ & 0.054 \\
\hline
\end{tabular}

${ }^{a}$ Data missing for 3 patients

${ }^{\mathrm{b}}$ Data missing for 1 patient

$* p=0.073$ (patients vs. control group 1)

${ }^{\#} \mathrm{p}=0.023$ (patients vs. control group 1)

${ }^{+} \mathrm{p}=0.046$ (patients vs. control group 1 )

${ }^{\ddagger} \mathrm{p}=0.10$ (patients vs. control group 1)

II $\mathrm{p}=0.11$ (patients vs. control group 1)

${ }^{\beta} \mathrm{p}=0.57$ (patients vs. control group 1)

$\gamma \mathrm{p}=0.025$ (patients vs. control group 1) 
Table II. HLA B8/DQ2, DR3/DQ2, B8/DR3 haplotype frequencies in patients and control groups.

\begin{tabular}{lcccc}
\hline HLA groups & Patients & Control group & Control group 2 & $\mathrm{p}$ \\
& $\mathrm{N}(\%)$ & $1 \mathrm{~N}(\%)$ & $\mathrm{N}(\%)$ & 0.001 \\
\hline HLA B8/ DQ2 & $25(35.7)^{\mathrm{a}^{*}}$ & $3(13.0)^{*}$ & $7(10.6)$ & $<0.001$ \\
HLA DR3/DQ2 & $53(72.6)$ & $15(65.2)^{\sharp}$ & $19(28.8)^{\sharp}$ & 0.001 \\
\hline
\end{tabular}

${ }^{a}$ Data missing for 3 patients

${ }^{*} \mathrm{p}=0.039$ (patients vs. control group 1$)$

" $\mathrm{p}=0.002$ (control group 1 vs. control group 2)

${ }^{+} \mathrm{p}=0.03$ (patients vs. control group 1 )

Table III. HLA antigens and risk of development of celiac disease.

\begin{tabular}{llll}
\hline HLA antigen & Odds ratio & $95 \%$ Confidence interval & $\mathrm{p}$ \\
\hline B8 & 4.5 & $1.88-10.99$ & 0.001 \\
B50 & 9.4 & $2.08-43.08$ & 0.004 \\
C6 & 2.8 & $1.23-6.18$ & 0.013 \\
C7 & 2.6 & $1.27-5.27$ & 0.008 \\
DQ2 & 9.8 & $4.05-23.5$ & $<0.001$ \\
DR3 & 7.2 & $3.38-15.47$ & $<0.001$ \\
\hline
\end{tabular}

control group 1. While there was no difference regarding HLA B8/DQ2 and HLA B8/DR3 haplotypes between 2 control groups, HLA DR3/DQ2 haplotype was significantly more common in control group 1 than control group 2.

Table III shows odds ratios for CD in different HLA types. The presence of DQ2 allele increased the risk of CD 9.8 times.

Thirty $(41.1 \%)$ of the patients presented with typical findings and 43 (58.9\%) with atypical findings. No silent disease was detected. When the relationship between disease presentation and HLA groups was evaluated, it was found that only HLA DQ6 allele was related with typical presentation.

Totally $13(17.8 \%)$ of the patients had another coexisting autoimmune disease; seven had type1 DM, six had autoimmune thyroiditis. When the relationship between HLA types and having another autoimmune disease was evaluated, no association was found between HLA type and having more than one autoimmune disease.
In the patient group, 15 families (20.5\%) had two or more celiac disease patients. It was determined that the individuals who had HLA A69, B41 and C12 alleles had significantly higher chance of having a relative suffering from $C D(p<0.05)$.

Histologic examination revealed that 5 (6.8\%) patients had Marsh 2, 25 (34.3\%) patients Marsh 3a, 28 (38.4\%) patients Marsh 3b, and $15(20.5 \%)$ patients had Marsh 3c changes. When the relationship between the severity of histopathologic changes with HLA groups was investigated, a positive correlation of HLA 15 allele with Marsh 3a lesion was found ( $\mathrm{p}$ $<0.047)$. No relation between histopathological impairment and any other allele was found.

In our study, we did not detect any relation between co-existence of $\mathrm{CD}$ and any other autoimmune disease and HLA antigens. Although, a higher risk of co-existence of other autoimmune diseases and CD with HLA DR4 antigen was detected, it did not reach statistical significance $(p=0.06)$. HLA DR7 tended to be more common among $C D$ patients without other autoimmune diseases $(p=0.08)$. 


\section{Discussion}

Genetic, environmental and immunological factors together play a role in the development of CD. HLA DQ2 and DQ8 are known as the most important genetic factors. ${ }^{1}$ Beside HLA DQ2/DQ8, the most common celiac disease related alleles are HLA DR3 and HLA B8. ${ }^{6-9}$ Our results show that in Turkey HLA DR3 and HLA B8 alleles are also frequent among celiac patients.

The studies investigating the relationship between CD and HLA alleles in the Turkish population are limited. ${ }^{10-14}$ When the results of these studies are examined, it is seen that HLA DQ2, DQ8, B8 alleles are risky alleles in the emerge of $C D$ in all studies. Apart from these alleles, some alleles may also be risky for the development of $\mathrm{CD}$. Some alleles may have preventative roles. These results are summarized in Table IV.

Our findings also confirmed that the most important genetic risk factor for $\mathrm{CD}$ is positivity of DQ2 (89\%). Considering that B8 and B50 are significantly higher in the patient group than the control group 1 , these alleles may be factors that facilitate the occurrence of the disease. However, it is still controversial as to whether these alleles predispose toward CD. Considering the studies performed in our country B8 antigen was reported to be highly positive among celiac patients. In some studies performed in Turkey and Europe DR3 was frequent and considered a disease-related allele among CD. ${ }^{10,15,16}$

Although there was a statistically significant difference regarding DR3 and C6 between the three groups, the difference between the patient group and control group was not significant in our study. There is no other study reporting HLA C6 allele frequency among Turkish celiac patients. Two studies performed in Turkey reported carrying HLA C7 allele as a risk factor for $\mathrm{CD}$, similar to our study. ${ }^{10,13}$

Although there are no other studies supporting the relationship of this allele with $\mathrm{CD}$, there is one study reporting that the presence of $\mathrm{C} 7$ may be related to the late presentation of $\mathrm{CD} .{ }^{17}$ It was reported that DR7 was also frequent among celiac patients similar to our study. ${ }^{10,13}$ Our results generally supported other studies performed either in Turkey or other countries. However, distinctly from other studies HLA B50 allele was detected more frequently among our patient group in comparison to control groups and HLA A25 and A2, which were considered as risk factors in other studies, were found to be similar in both groups (data not given). With these results we suggest that HLA B8, B50, and DR7 may be factors that increase the effect of the presence of DQ2 on the development of CD among Turkish people.

There are only a few studies about the protective role of some alleles against $\mathrm{CD}$ and there is no consensus on this subject. Ruiz del Prado et al. ${ }^{18}$ noted that $\mathrm{B} 8, \mathrm{C} 7, \mathrm{DR} 3, \mathrm{DR} 7$ and DQ2 are more prevalent among patients while C4 and DQ1 are prevalent in the general population and these can be protective from the disease. A study from our country reported that HLA A29, B51, C5, DQ1, DR14, DR15, and DR16 alleles to be more prevalent in the general population compared to patient groups. ${ }^{13}$ In this study HLA B27, C14, DR11 and DQ3 alleles were less frequent among patients, compared to control groups, and HLA A30 tended to be lower, although statistically non-significant. The HLA DR15 allele was rare

Table IV. Risky and protective HLA alleles in Turkish children with celiac disease.

\begin{tabular}{lcrll}
\hline Authors & Year & Number (N) Risky alleles (HLA) & Protective alleles (HLA) \\
\hline Tümer et al. ${ }^{10}$ & 2000 & 33 & DQ2, B8, A2, C7, DR3, DR7, DR3/ & \\
& & & DR4, DR3/DQ2, DR7/DQ2 & \\
${\text { Erkan et al. }{ }^{12}}^{2} 1999$ & 30 & DQ2, B8, A25, DR18 & B4 \\
Kuloğlu et al. ${ }^{13}$ & 2008 & 75 & DQ2, B8, B13, C7, DR7, DR17 & \\
Present study & & 73 & DQ2, B8, B50, C6, C7, DR3, DR7 & DQ3, A30, B27, C14, DR11, DR15 \\
\hline
\end{tabular}


in patients and their relatives compared to the general population. The frequencies of alleles detected in the control group in our study were not compatible with this literature except DR15 that might be protective.

Genetic factors affecting the clinical presentation of the disease has been claimed by some researchers. It was stated that individuals with HLA B8 and C7 presented with the disease in elder ages. ${ }^{17}$ The relation of disease presentation with HLA B8 and DR3 was noted as well. ${ }^{19}$ Majorana et al. ${ }^{20}$ reported that patients with tooth enamel disorder carried HLA DR3 genotype with a higher incidence. There is only one study stating the correlation between HLA and disease presentation in the Turkish population. In this study consisting of 75 patients (40 with typical and 35 with atypical presentation), it was reported that HLA B35, DR11, and DQ7 antigens were related to typical and HLA B8 antigen with atypical presentation. ${ }^{13}$ In this study only HLA DQ6 related to typical presentation was found; but no relation with the others was obtained.

Genetic factors have been suggested to play a role in the development autoimmune diseases such as type $1 \mathrm{DM}$, autoimmune thyroiditis and Addison disease and this relation has been basically ascribed to major HLA antigens. ${ }^{2,3}$ Recent studies have shown some HLA antigens to be related to the tendency for these disorders while some others having protective effects. ${ }^{13,21}$ Britten etal. ${ }^{22}$ investigated the relation of HLA DQ gene expression and susceptibility to diseases especially to type1 DM. They reported that DQ2 and DQ8 antigens to be related to increase in disease risk. HLA DR3 positivity was found to be higher among patients who have type $1 \mathrm{DM}$ and $\mathrm{CD}$ together compared to the normal population and patients with only type $1 \mathrm{DM}^{23}$ Baker et $\mathrm{al}^{24}$ noted that HLA DQ2 and DQ8 alleles were the genetic reasons for autoimmune disease as well as CD. In another study, in patients with type1 DM, T-cell activity against wheat antigens causing intestinal inflammation were not related to DQ2 allele but to DR4 allele. ${ }^{25}$ It was also reported that DR4 haplotypes such as DR4/DQ8 could be associated with CD susceptibility. ${ }^{26}$ In our study we did not detect any relation between co-existence of celiac disease and other autoimmune diseases and HLA antigens. However, a higher risk of co-existence of other autoimmune diseases and celiac disease with HLA DR4 antigen was detected, not reaching statistical significance $(p=0.06)$. HLA DR7 was prevalent among $\mathrm{CD}$ patients without other autoimmune diseases again not reaching statistical significance $(\mathrm{p}=0.08)$. It will be easier to comment on these antigens' roles in studies with higher patient numbers.

It was shown that the incidence of CD among European populations was similar to that of immigrants with European ancestry in USA and Australia. ${ }^{27}$ It was previously reported that having a relative with $C D$, especially having a sibling, was the most important risk factor for the disease, raising the risk up to $20 \%$. Besides, the risk was found to be the highest in HLA DQ2 positive male patients in a family having two affected siblings. It was also reported that the asymptomatic relatives of patients with CD could have either intestinal mucosal structural deterioration or functional alterations of permeability. It was also stated that the risk of other autoimmune diseases and intestinal lymphoma was increased in these asymptomatic relatives. ${ }^{27}$ A study from an Asian population indicated the incidence of $\mathrm{CD}$ in relatives of celiac disease patients to be $4.4 \%$ and the rate of relatives negative for HLA DQ2/DQ8 was found as $15 \%{ }^{28}$ In our study we observed the frequency of alleles (DQ2/ DR3) causing predisposition to celiac disease in healthy siblings of patients was higher than the normal population. However, no correlation of these alleles and celiac disease occurrence in more than one patient in the same family was detected. There was an increased frequency of HLA A69, B41 and C12 alleles in patients with relatives having $C D$ in their families. There are no previous studies investigating CD and HLA antigen relation in families with high number of patients. In this respect we believe our study 
is contributing to this new area. The results should be tested in studies with larger numbers of subjects.

The factors related to the damage of small intestinal mucosa in $\mathrm{CD}$ has not been well clarified. There are studies pointing to the relation between the degree of mucosal damage and HLA antigens. Jores et al. ${ }^{29}$ stated that the histo-pathological damage was more severe in patients homozygous for HLA DQB1*0201 allele. Karinen et al. ${ }^{30}$ reported that HLA DQB $1{ }^{*} 0201$ allele was related to more severe disease, late histological amelioration and early onset of disease. Another study indicated that CD patients homozygous for HLA DQ2 alleles should be diagnosed at early stages of the disease because of higher rates of intestinal $\mathrm{T}$ cell lymphoma development. ${ }^{31} \mathrm{~A}$ total of 23 patients in our study group was homozygous for DQ2 allele but no relevance to the degree of histopathological damage was detected. Simone et al. ${ }^{32}$ emphasized the strong relation between soluble cytotoxic T lymphocyte related protein-4 (sCTLA-4) gene and autoimmune disease development and also stated that sCTLA-4 was higher in CD patients without treatment and those levels correlated to antitissue transglutaminase antibody levels and the degree of intestinal mucosal damage. No relationship between HLA antigens and sCTLA-4 levels were detected. We detected that histological damage was significantly severe in CD patients with HLA B8 antigen. We could not find any study investigating the relation between HLA B8 antigen and the severity of damage in the small bowel. However, HLA B8 antigen was reported to be more frequent in Europe and in our country. ${ }^{10,13,19}$ There are studies claiming that this antigen has a relationship with disease tendency, disease presentation and age of disease onset. ${ }^{7-10,17,19}$ In our study, it was also found that HLA DR3 and HLA B8 alleles were common besides HLA DQ2 allele. However, no relationship was found between HLA DQ2 and DR3 alleles and the severity of intestinal mucosal damage. In patients with DR15 allele the mucosal damage was lower. There is no data disclosing the relationship between mucosal damage and DR15 in the literature.

In conclusion, HLA DQ2, B8, B50, C6, C7, DR3 and DR7 were identified to be risky alleles for the development of $\mathrm{CD}$ in the Turkish population while HLA B27, C14, DR11, DR15, DQ3 alleles, and partly HLA A30 could be protective. DQ8 allele was not found in either the celiac or control groups. HLA DQ allele was found to be related to typical presentation, and HLA DR15 allele was predictive of mild mucosal damage.

\section{REFERENCES}

1. NIH Consensus Statement on Celiac Disease. NIH Consens State Sci Statements 2004; 21: 1-23.

2. Lebwohl B, Sanders DS, Green PHR. Coeliac disease. Lancet 2018; 391: 70-81.

3. Brown JRG, Singh P. Coeliac disease. Paediatr Int Child Health 2019; 39: 23-31.

4. Hill ID, Dirks MH, Liptak GS, et al; North American Society for Pediatric Gastroenterology, Hepatology and Nutrition. Guideline for diagnosis and treatment of celiac disease in children: Recommendations of the North American Society for Pediatric Gastroenterology, Hepatology and Nutrition. J Pediatr Gastroenterol Nutr 2005; 40: 1-19.

5. Revised criteria for diagnosis of coeliac disease. Report of Working Group of European Society of Paediatric Gastroenterology and Nutrition. Arch Dis Child 1990; 65: 909-911.

6. Ryan AW, Thornton JM, Brophy $K$, et al. Chromosome $5 \mathrm{q}$ candidate genes in coeliac disease: genetic variation at IL4, IL5, IL9, IL13, IL17B and NR3C1. Tissue Antigens 2005; 65: 150-155.

7. Bolognesi E, Karell K, Percopo S, et al. Additional factor in some HLA DR3/DQ2 haplotypes confers a fourfold increased genetic risk of celiac disease. Tissue Antigens 2003; 61: 308-316.

8. Louka AS, Sollid LM. HLA in coeliac disease: unravelling the complex genetics of a complex disorder. Tissue Antigens 2003; 61: 105-117.

9. Louka AS, Moodie SJ, Karell K, et al; European Genetics Cluster on Celiac Disease. A collaborative European search for non-DQA1*05-DQB1*02 celiac disease loci on HLA-DR3 haplotypes: analysis of transmission from homozygous parents. Hum Immunol 2003; 64: 350-358. 
10. Tümer L, Altuntaş B, Hasanoglu A, Söylemezoglu $\mathrm{O}$, Arinsoy T. Pattern of human leukocyte antigens in Turkish children with celiac disease. Pediatr Int 2000; 42: 678-681.

11. Tüysüz B, Dursun A, Kutlu T, et al. HLA-DQ alleles in patients with celiac disease in Turkey. Tissue Antigens 2001; 57: 540-542.

12. Erkan T, Kutlu T, Yılmaz E, Cullu F, Tümay T. Human leukocyte antigens in Turkish pediatric celiac patients. Turk J Pediatr 1999; 41: 181-188.

13. Kuloğlu Z, Doğancı T, Kansu A, et al. HLA types in Turkish children with celiac disease. Turk J Pediatr 2008; 50: 515-520.

14. Basturk A, Artan R, Yilmaz A. The incidence of HLADQ2/DQ8 in Turkish children with celiac disease and a comparison of the geographical distribution of HLA-DQ. Prz Gastroenterol 2017; 12: 256-261.

15. Megiorni F, Mora B, Bonamico M, et al. HLA-DQ and susceptibility to celiac disease: evidence for gender differences and parent-of-origin effects. Am J Gastroenterol 2008; 103: 997-1003.

16. Donat E, Planelles D, Capilla-Villanueva A, Montoro JA, Palau F, Ribes-Koninckx C. Allelic distribution and the effect of haplotype combination for HLA type II loci in the celiac disease population of the Valencian community (Spain). Tissue Antigens 2009; 73: 255-261.

17. Vogelsang H, Panzer S, Mayr WR, Granditsch G, Fischer GF. Distribution of HLA class I alleles differs in celiac disease patients according to age of onset. Dig Dis Sci 2003; 48: 611-614.

18. Ruiz del Prado MY, Olivares Lopez JL, Lazaro Almarza A, Lasierra Díaz MP. HLA system. Phenotypic and gene frequencies in celiac and healthy subjects from the same geographical area. Rev Esp Enferm Dig 2001; 93: 106-113.

19. Lopez-Vazquez A, Fuentes D, Rodrigo L, et al. MHC class I region plays a role in the development of diverse clinical forms of celiac disease in a Saharawi population. Am J Gastroenterol 2004; 99: 662-667.

20. Majorana A, Bardellini E, Ravelli A, Plebani A, Polimeni A, Campus G. Implications of gluten exposure period, CD clinical forms, and HLA typing in the association between celiac disease and dental enamel defects in children. A case-control study. Int J Paediatr Dent 2010; 20: 119-124.

21. Sumnik Z, Kolouskova S, Malcova H, et al. High prevalence of coeliac disease in siblings of children with type 1 diabetes. Eur J Pediatr 2005; 164: 9-12.
22. Britten AC, Mijovic CH, Barnett AH, Kelly MA Differential expression of HLA-DQ alleles in peripheral blood mononuclear cells: alleles associated with susceptibility to and protection from autoimmune type 1 diabetes. Int J Immunogenet 2009; 36: 47-57.

23. Kahaly GJ, Frommer L, Schuppan D. Celiac disease and endocrine autoimmunity - the genetic link. Autoimmun Rev 2018; 17: 1169-1175.

24. Baker PR, Baschal EE, Fain PR, et al. Haplotype analysis discriminates genetic risk for DR3associated endocrine autoimmunity and helps define extreme risk for Addison's disease. J Clin Endocrinol Metab 2010; 95: E263-E270.

25. Mojibian M, Chakir H, Lefebvre DE, et al. Diabetesspecific HLA-DR-restricted proinflammatory T-cell response to wheat polypeptides in tissue transglutaminase antibody-negative patients with type 1 diabetes. Diabetes 2009; 58: 1789-1796.

26. Spurkland A, Sollid LM, Polanco I, Vartdal F, Thorsby E. HLA -DR and -DQ genotypes of celiac disease patients serologically typed to be non-DR3 or non-DR5/7. Hum Immunol 1992; 35: 188-192.

27. Freeman HJ. Risk factors in familial forms of celiac disease. World J Gastroenterol 2010; 16: 1828-1831.

28. Srivastava A, Yachha SK, Mathias A, Parveen F, Poddar U, Agrawal S. Prevalence, human leukocyte antigen typing and strategy for screening among Asian first-degree relatives of children with celiac disease. J Gastroenterol Hepatol 2010; 25: 319-324.

29. Jores RD, Frau F, Cucca F, et al. HLA-DQB1* 0201 homozygosis predisposes to severe intestinal damage in celiac disease. Scand J Gastroenterol 2007; 42: 48-53.

30. Karinen H, Kärkkäinen $P$, Pihlajamäki J, et al. HLA genotyping is useful in the evaluation of the risk for coeliac disease in the 1st-degree relatives of patients with coeliac disease. Scand J Gastroenterol 2006; 41: 1299-1304.

31. Al-Toma A, Goerres MS, Meijer JW, Peña AS, Crusius JB, Mulder CJ. Human leukocyte antigenDQ2 homozygosity and the development of refractory celiac disease and enteropathy-associated T-cell lymphoma. Clin Gastroenterol Hepatol 2006; 4: 315-319.

32. Simone R, Brizzolara R, Chiappori A, et al. A functional soluble form of CTLA-4 is present in the serum of celiac patients and correlates with mucosal injury. Int Immunol 2009; 21: 1037-1045. 